\title{
Control and Cancellation Singularities of Bilaplacian in a Cracked Domains
}

\author{
Gilbert Bayili ${ }^{1}$, Cheikh Seck ${ }^{2}$, Abdoulaye Sène $^{3} \&$ Mary Teuw Niane ${ }^{2}$ \\ ${ }^{1}$ LAME, Université de Ouagadougou, Burkina Faso \\ ${ }^{2}$ LANI, UGB, Saint-Louis, Sénégal \\ ${ }^{3}$ UMI 209 UMMISCO, UCAD, Dakar, Sénégal \\ Correspondence: Cheikh, Seck, LANI, UGB, Saint-Louis, Sénégal. E-mail: cseck2003@yahoo.fr \\ Received: January 4, 2012 Accepted: January 19, 2012 Online Published: July 4, 2012 \\ doi:10.5539/jmr.v4n4p35 URL: http://dx.doi.org/10.5539/jmr.v4n4p35
}

\begin{abstract}
We are interested in controlling and removing singularities of the Dirichlet problem involving the bilaplacian operator in a domain with corner. It's possible of making the solution to the bilaplacian operator regular, through acting on a small part of a cracked domain with corner. Then, the best singularity coefficients can be controlled by simultaneous actions of two controls on a small part of the boundary.
\end{abstract}

Keywords: Bilaplacian, singular functions, dual singular functions, cracks

\section{Introduction and Statement of Problem}

We consider the Dirichlet problem for the bilaplacian operator in a bounded polygonal domain $\Omega$ of $\mathbb{R}^{2}$. Since the domain is polygonal, the solution of this problem does not only depend on the regularity of data, but also on the geometry of the domain (Grisvard, P., 1974; Grisvard, P., 1992; Kondratiev, V. A., 1967). This solution is singular in the neighbourhood of non-convex vertices of $\Omega$ (see Bayili, G., 2009; Seck, C., Bayili, G., Sène, A., \& Niane, M. T., 2011). Niane et al. (2006) proved that it is possible by acting on a small part of the domain or on a small part of the borders, a regular solution of the Laplace equation can be obtained. Let $m+1$ the number of non-convex angles of $\Omega$ and $\bar{O}$ a non empty open bounded $\Omega$. We will show that there are infinitely differentiable functions with support in $\bar{O}$ and satisfying the following condition if $f \in L^{2}(\Omega),\left(\lambda_{i}\right)_{1 \leq i \leq k}$ are the coefficients of the singularities and $\left(g_{i}\right)_{1 \leq i \leq k}$ the singularities of the problem

$$
\left\{\begin{array}{c}
\text { Find } v \in H_{0}^{2}(\Omega) \text { such that } \\
-\Delta^{2} v=f \text { in } \Omega
\end{array}\right.
$$

then the problem

$$
\left\{\begin{array}{l}
\text { Find } y \in H_{0}^{2}(\Omega) \text { such that } \\
-\Delta^{2} y=f-\sum_{i=1}^{k} \lambda_{i} g_{i} \text { in } \Omega
\end{array}\right.
$$

has an unique solution $y \in H^{4}(\Omega)$.

We will also prove the following result if $\Gamma_{1}$ and $\Gamma_{2}$ are two analytical open sets of $\Gamma$ whose measure of the intersection is non zero, then there exist $k$ functions $\left(h_{i}, g_{i}\right)_{1 \leq i \leq k}$ of $D\left(\Gamma_{1}\right) \times D\left(\Gamma_{2}\right)$ with compact support contained in $\Gamma_{1} \cap \Gamma_{2}$ such that

$$
\left\{\begin{array}{c}
-\Delta^{2} y=f \text { in } \Omega \\
\gamma y=-\sum_{i=1}^{k} \lambda_{i} h_{i} \text { on } \Gamma_{1} \\
\frac{\partial y}{\partial v}=\sum_{i=1}^{k} \lambda_{i} g_{i} \text { on } \Gamma_{2}
\end{array}\right.
$$

has an unique solution $y \in H^{4}(\Omega)$. 


\section{Bi-orthogonality Property of Biharmonic Functions}

Let $H$ be a Hilbert space with a scalar product $\langle\cdot, \cdot\rangle_{H}$.

Lemma (Density lemma) Let $H$ be a Hilbert space, $D$ a dense subspace in $H$ and $\left\{e_{0}, \ldots, e_{m}\right\}$ a subset of $H$. Then, there exist $\left\{d_{0}, \ldots, d_{m}\right\}$ in $D$ such that $\forall 1 \leq i<j \leq m, \quad\left\langle e_{i}, d_{j}\right\rangle_{H}=\delta_{i j}$.

Proof. According to the hypothesis and by Gram-Schmidt orthogonalization, there exist $v_{0}, \ldots, v_{m}$, such that $\left\langle v_{i}, e_{j}\right\rangle_{H}=\delta_{i j}, \forall 1 \leq i<j \leq m$. As $D$ is dense in $H$, there exist sequences $\left(v_{i}^{(n)}\right)$ of elements in $D$, such that $v_{i}^{(n)} \longrightarrow v_{i}$ in $H$ as $n \longrightarrow \infty$, for all $i \in\{0, \ldots, m\}$. This implies that $\left\langle v_{i}^{(n)}, e_{j}\right\rangle_{H} \longrightarrow\left\langle v_{i}, e_{j}\right\rangle_{H}=\delta_{i j}$ as $n \longrightarrow \infty$, and hence the matrix $\mathcal{K}_{n}=\left(\left\langle v_{i}^{(n)}, e_{j}\right\rangle_{H}\right)_{0 \leq i<j \leq m}$ is invertible for $n$ large enough. Fixed this value of $n$, write $\mathcal{K}_{n}^{-1}=\left(c_{i j}\right)_{0 \leq i<j \leq m}$. The requested elements are $d_{i}=\sum_{k=0}^{m} c_{i k} v_{k}^{(n)}$, since $\left\langle d_{i}, e_{j}\right\rangle_{H}=\sum_{k=0}^{m} c_{i k}\left\langle v_{k}^{(n)}, e_{j}\right\rangle_{H}=\delta_{i j}$.

Theorem Let $\Omega$ be an open set of $\mathbb{R}^{n}$ and $O$ a non-empty open set of $\Omega$. If $\left(\omega_{i}\right)_{1 \leq i \leq k}$ is a set of linearly independent of biharmonic functions of $L^{2}(\Omega)$, then there exists a family $\left(g_{j}\right)_{1 \leq j \leq k}$ of $C^{\infty}$ functions with compact support in $\bar{O}$, such that

$$
\forall 0 \leq j<i \leq k \text {, we have } \int_{\Omega} \omega_{i} g_{j} d x=\delta_{i j}
$$

Proof. Let $H=L^{2}(\bar{O})$. The family $\left(\omega_{i \mid \bar{O}}\right)_{1 \leq i \leq k}$ is linearly independent ?

Effectively, assume that there exist real numbers $\left(\alpha_{i}\right)_{1 \leq i \leq k}$ not all of them zero such that

$$
\sum_{i=1}^{k} \alpha_{i} \omega_{i}=0 \text { in } \bar{O}
$$

We know that $\sum_{i=1}^{k} \alpha_{i} \omega_{i}$ is an analytical form, according to the unicity theorem of Holmgren's-Kovalevska in L. Hormander (1976), we have $\sum_{i=1}^{k} \alpha_{i} \omega_{i}=0$ on $\Omega$, we can deduce by hypothesis that $\alpha_{i}=0, \forall i \in\{1, \ldots, k\}$ and consequently $\left(\omega_{i \mid \bar{O}}\right)_{1 \leq i \leq k}$ is linearly independent.

Since $D(\bar{O})$ is dense in $L^{2}(\bar{O})$, Niane et al. (2006) and Density Lemma imply that there exists a family $\left(g_{j}\right)_{1 \leq j \leq k}$ of functions of $D(\Omega)$ with support in $\bar{O}$ such that:

$$
\forall 0 \leq j<i \leq k, \int_{\Omega} \omega_{i} g_{j} d x=\delta_{i j}
$$

Theorem Let $\Omega$ be a non-empty bounded open polygon with $\mathbb{R}^{n}$ of boundary $\Gamma$. Let $\Gamma_{1}$ and $\Gamma_{2}$ be two non-empty analytic open sets of $\Gamma$ such that mes $\left(\Gamma_{1} \cap \Gamma_{2}\right) \neq 0$. Let $\left(\omega_{i}\right)_{1 \leq i \leq k}$ be a linear independent family of biharmonic functions of $L^{2}(\Omega)$ verifying

$$
\omega_{i}=\frac{\partial \omega_{i}}{\partial v}=0 \text { on } \Gamma \text { and }\left(\gamma \frac{\partial \Delta \omega_{i}}{\partial v}\left|\Gamma_{1}, \gamma \Delta \omega_{i}\right| \Gamma_{2}\right) \in L^{2}\left(\Gamma_{1}\right) \times L^{2}\left(\Gamma_{2}\right),
$$

then there exist $k$ functions $\left(h_{i}, g_{i}\right)_{1 \leq i \leq k}$ of $D\left(\Gamma_{1}\right) \times D\left(\Gamma_{2}\right)$ with compact support contained in $\Gamma_{1} \cap \Gamma_{2}$ verifying

$$
\forall 0 \leq j<i \leq k, \int_{\Gamma}\left(\Delta \omega_{i} g_{j}+\frac{\partial \Delta \omega_{i}}{\partial v} h_{j}\right) d \sigma=\delta_{i j} .
$$

Proof. Let the space $H=L^{2}\left(\Gamma_{1}\right) \times L^{2}\left(\Gamma_{2}\right)$ with the following scalar product

$$
\forall\left(x_{1}, y_{1}\right),\left(x_{2}, y_{2}\right) \in H,\left\langle\left(x_{1}, y_{1}\right),\left(x_{2}, y_{2}\right)\right\rangle=\left\langle x_{1}, x_{2}\right\rangle+\left\langle y_{1}, y_{2}\right\rangle
$$

With this product, $H$ is a Hilbert space. Next we prove that the family $\left\{\left(\left.\frac{\partial \Delta \omega_{i}}{\partial v}\right|_{\Gamma_{1}},\left.\Delta \omega_{i}\right|_{\Gamma_{2}}\right)_{1 \leq i \leq k}\right\}$ is linearly independent.

Assume the existence of real numbers $\left(\alpha_{i}\right)$ such that

$$
\sum_{i=1}^{k} \alpha_{i}\left(\left.\frac{\partial \Delta \omega_{i}}{\partial v}\right|_{\Gamma_{1}},\left.\Delta \omega_{i}\right|_{\Gamma_{2}}\right)_{1 \leq i \leq k}=0
$$

This implies that

$$
\left\{\begin{array}{c}
\left.\sum_{i=1}^{k} \alpha_{i} \frac{\partial \Delta \omega_{i}}{\partial v}\right|_{\Gamma_{1}}=0 \\
\sum_{i=1}^{k} \alpha_{i}\left(\left.\Delta \omega_{i}\right|_{\Gamma_{2}}\right)=0
\end{array}\right.
$$


Since $\psi=\sum_{i=1}^{k} \alpha_{i} \omega_{i}$ is an analytical form in virtue of the Holmgren and the Cauchy-Kovalevska theorem (see Hormander, L., 1976). Hence, we can deduce under our hypothesis that

$$
\left\{\begin{array}{c}
\Delta^{2} \psi=0 \text { in } \Omega \\
\psi=\frac{\partial \psi}{\partial \nu}=0 \text { on } \Gamma \\
\frac{\partial \Delta \psi}{\partial v}=0 \text { on } \Gamma_{1} \\
\Delta \psi=0 \text { on } \Gamma_{2}
\end{array}\right.
$$

According to the Cauchy-Kowalevska Theorem, there exists a non-empty open neighbourhood $O \subset \Gamma_{1} \cap \Gamma_{2}$ such that $\sum_{i=1}^{k} \alpha_{i} \omega_{i}=0$ in $O$. By Holmgren Theorem (Hormander, L., 1976), we obtain:

$$
\sum_{i=1}^{k} \alpha_{i} \omega_{i}=0 \text { in } O
$$

Consequently, we have:

$$
\sum_{i=1}^{k} \alpha_{i} \omega_{i}=0 \text { in } \Omega
$$

So we can deduce that $\alpha_{i}=0, \forall i$ and the family

$$
\left(\left.\frac{\partial \Delta \omega_{i}}{\partial v}\right|_{\Gamma_{1}},\left.\Delta \omega_{i}\right|_{\Gamma_{2}}\right)_{1 \leq i \leq k}
$$

is linearly independent.

Since $D\left(\Gamma_{1}\right) \times D\left(\Gamma_{2}\right)$ is dense in $L^{2}\left(\Gamma_{1}\right) \times L^{2}\left(\Gamma_{2}\right)$, Niane et al. (2006) proved the existence of a family $\left(h_{i}, g_{i}\right)_{1 \leq i \leq k}$ of compact support contained in $\Gamma_{1} \cap \Gamma_{2}$ such that

$$
\forall 0 \leq j<i \leq k, \int_{\Gamma}\left(\Delta \omega_{i} g_{j}+\frac{\partial \Delta \omega_{i}}{\partial v} h_{j}\right) d \sigma=\delta_{i j}
$$

\section{Cancellation of Singularities}

\subsection{Preliminary Results on Dual Singular Functions}

We show that, in a cracked domain, we can obtain a regular solution of the biharmonic problem by acting two simultaneous controls on two small parts of the boundary of intersection not empty and not reduce to a point on the small part $O$ of $\Omega$ not intercepting any vertices.

Lemma (P. Grisvard, 1985) If $f \in L^{2}(\Omega)$, the solution $u$ of Problem (1) related to the crack $O_{i}$ is writen as $u=u_{R}+\sum_{i=1}^{4} \lambda_{i} S_{i}$ where $u_{R} \in H^{4}(\Omega)$ and $\lambda_{i} \in \mathbb{R}$ for $i \in\{1, \ldots, 4\}$. This singular part is described below by its polar coordinates

$$
S_{i}(r, \theta)=r^{\alpha_{i}} \sin \left(\alpha_{i} \theta\right) \eta_{i}(r)
$$

where $\alpha_{i}=\frac{\pi}{\omega_{i}}$ is the singularity exponent related to the crack $O_{i}$ and $\eta_{i}$ is cut-off function equal to 1 on the neighbourhood of vertex of the open $O_{i}$.

By Grisvard Lemma, in each non-convex vertices, we have finite number of dual singular solutions associated with the domain $\Omega$.

Pose $\omega_{i}^{*}=r^{-j} S_{i}(r, \theta)=r^{\alpha_{i}-j} \sin \left(\alpha_{i} \theta\right) \eta_{i}(r)$ for $1 \leq i \leq k$ and $j \in\{1,3\}$. According to Grisvard $(1985 ; 1989)$ and Timouyas (2003), $\left(\omega_{i}^{*}\right)_{1 \leq i \leq k}$ is the family of dual singular solutions associated to $m$ angles of non-convex vertex of domain $\Omega$. This family is linearly independent and verifies

$$
\forall i \in\{1, \ldots, k\}, \omega_{i}^{*} \in L^{2}(\Omega) \cap V_{i}^{c} \text { and }\left\{\begin{array}{c}
\Delta^{2} \omega_{i}^{*}=0 \text { in } \Omega \\
\gamma \frac{\partial \omega_{i}^{*}}{\partial v}=\gamma \omega_{i}^{*}=0 \text { in } \Gamma
\end{array}\right.
$$


with $V_{i}$ the $i^{\text {th }}$ open neighbourhood of vertex of $O_{i}$ of the domain $\Omega$.

The singularity coefficients $\left(\lambda_{i}\right)_{1 \leq i \leq k}$ associeted with problem

$$
\left\{\begin{array}{c}
\text { Find } u \in H_{0}^{2}(\Omega), \text { such that } \\
\forall v \in H_{0}^{2}(\Omega): \int_{\Omega} \Delta u \Delta v d x=\int_{\Omega} f v d x
\end{array}\right.
$$

are obtained as

$$
\lambda_{i}=\int_{\Omega} f \omega_{i}^{*} d x
$$

\subsection{Cancellations of Singularities}

Theorem It exist $k$ infinitely differentiable functions with compact support contained in $\bar{O}$ such that if $f \in L^{2}(\Omega)$ and $\left(\lambda_{i}\right)_{1 \leq i \leq k}$ the singularity coefficients corresponding to the problem

$$
\left\{\begin{array}{c}
\Delta^{2} u=f \text { in } \Omega \\
\gamma u=\frac{\partial u}{\partial v}=0 \text { on } \Gamma
\end{array}\right.
$$

then the solution of problem

$$
\left\{\begin{array}{c}
\Delta^{2} \varphi=f-\sum_{i=1}^{k} \lambda_{i} g_{i} \text { in } \Omega \\
\gamma \varphi=\frac{\partial \varphi}{\partial \nu}=0 \text { on } \Gamma
\end{array}\right.
$$

verifies $\varphi \in H^{4}(\Omega) \cap H_{0}^{2}(\Omega)$.

Proof. The dual singular solutions of (16) verifies hypotheses of Theorem 2.2. Hence it exist a family $\left(g_{i}\right)_{1 \leq i \leq k}$ of functions with compact support contained in $\bar{O}$ such that

$$
\forall 0 \leq i<j \leq k, \int_{\Omega} \omega_{i} g_{j} d x=\delta_{i j}
$$

Let $\left(\lambda_{i}\right)_{1 \leq i \leq k}$ the singularity coefficients associeted with (15) and $\left(\zeta_{i}\right)_{1 \leq i \leq k}$ the singularity coefficients of (16). So we have:

$$
\begin{aligned}
\zeta_{i} & =\int_{\Omega} \omega_{i}^{*} \Delta^{2} \varphi d x=\int_{\Omega} \omega_{i}^{*}\left(f-\sum_{l=1}^{k} \lambda_{l} g_{l}\right) d x \\
& =\int_{\Omega} \omega_{i}^{*} f d x-\sum_{l=1}^{k} \lambda_{l} \int_{\Omega} \omega_{i}^{*} g_{l} d x=\lambda_{i}-\sum_{l=1}^{k} \lambda_{l} \delta_{i l}=\lambda_{i}-\lambda_{i}=0
\end{aligned}
$$

$\zeta_{i}=0$. Consequently and the solution is $\varphi \in H^{4}(\Omega) \cap H_{0}^{2}(\Omega)$

\section{Acknowledgment}

The authors would like to thank the Referee for his comments and suggestions. 


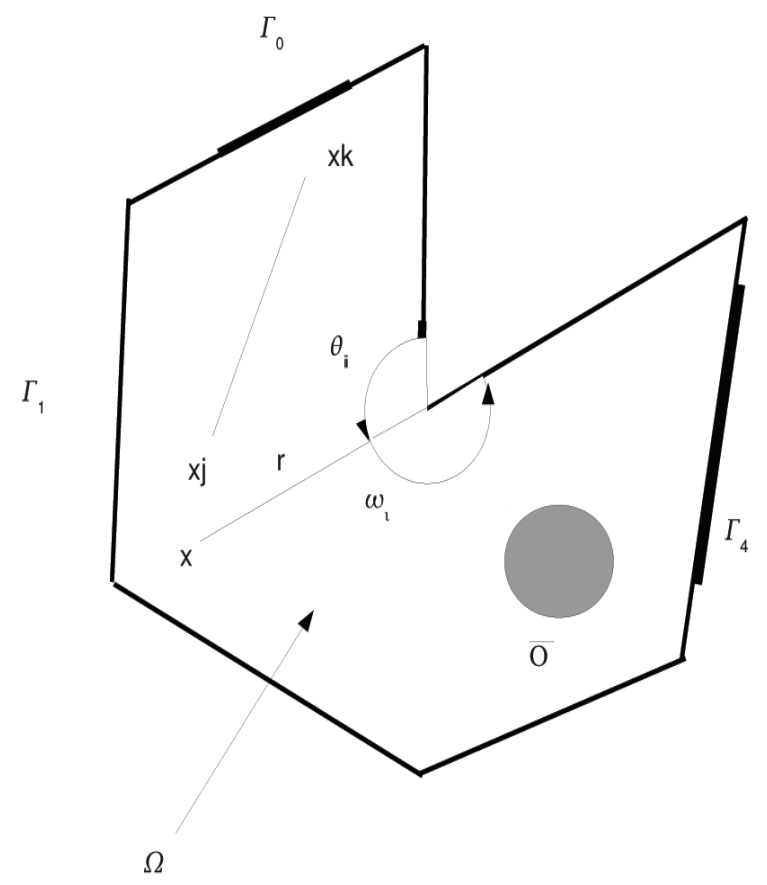

Figure 1. Non-convex cracked domain

\section{References}

Bayili, G. (2009). Contrôle des coefficients de singularités et contrôlabilité exacte dans un domaine polygonal avec fissure. Thèse unique, Université de Ouagadougou, Burkina Faso.

Grisvard, P. (1974). Problème de dirichlet dans un domaine non régulier. C. R. Acad. Sci. Paris., 278, Série A, $1615-1617$.

Grisvard, P. (1985). Elliptic problems in nonsmooth domains. Vol. 24 of Monographs and Studies in Mathematics. Pitman (Advanced Publishing Program), Boston, MA.

Grisvard, P. (1989). Contrôlabilité exacte des solutions de l'équation des ondes en présence de singularités. $J$. Maths. Pures et Appl., 215-259.

Grisvard, P. (1992). Singularities in boundary value problems. Research Notes in Applied Mathematics. Series Edition: P. G. Ciarlet and J.-L. Lions. Springer-Verlag.

Hormander, L. (1976). Linear partial differential operateurs. Springer-Verlag.

Kondratiev, V. A. (1967). Boundary value problems for elliptic equation in domain with conical or angular points. Transactions Moscow Mat. Soc., 227-313.

Mary Teuw Niane, Gilbert Bayili, Abdoulaye Sène, Abdou Sène, \& Mamadou Sy. (2006). Is it possible to cancel singularities in a domain with corners and cracks? C. R., Math., Acad. Sci. Paris, 343(2), 115-118. http://dx.doi.org/10.1016/j.crma.2006.05.003

Seck, C., Bayili, G., Séne, A., \& Niane, M. T. (2011). Contrôlabilité exacte de l'équation des ondes dans des espaces de sobolev non réguliers pour un ouvert poygonal. Afrika Matematika, 21(11).

Timouyas, H. (2003). Analyse et analyse numérique des singularités en électromagnétisme. Ph. D. thesis, Ecole Centrale de Lyon. 